\title{
Germanica
}

\section{Der Snob, de Sternheim (1913) : une comédie historique}

Sternheims Der Snob (1913) : eine historische Komödie.

Sternheim's Der Snob (1913): a historical Comedy.

Jean-Claude François

\section{OpenEdition}

\section{Journals}

Édition électronique

URL : http://journals.openedition.org/germanica/1365

DOI : 10.4000/germanica.1365

ISSN : 2107-0784

Éditeur

Université de Lille

Édition imprimée

Date de publication : 31 décembre 2011

Pagination : 11-22

ISBN : 9782913857285

ISSN : 0984-2632

\section{Référence électronique}

Jean-Claude François, "Der Snob, de Sternheim (1913) : une comédie historique », Germanica [En ligne], 49 | 2011, mis en ligne le 19 mars 2012, consulté le 06 octobre 2020. URL : http:// journals.openedition.org/germanica/1365; DOI : https://doi.org/10.4000/germanica.1365

Ce document a été généré automatiquement le 6 octobre 2020.

(c) Tous droits réservés 


\title{
Der Snob, de Sternheim (1913) : une comédie historique
}

\author{
Sternheims Der Snob (1913) : eine historische Komödie. \\ Sternheim's Der Snob (1913): a historical Comedy.
}

Jean-Claude François

1 Impossible de comprendre la charge comique de la pièce de Carl Sternheim, justement nommée "eine Komödie", sans envisager l'ensemble des quatre pièces qui se nourrissent de la vie d'une famille : les Maske. Une tétralogie donc qui commence avec Die Hose (1909-1910) et s'épanouit dans Der Snob (1913-1314) puis 1913 (1914) et enfin Das Fossil (1921-1922)1. Celui qui tient le tout, le fil conducteur, c'est Christian Maske, dépeint comme étant « le snob » par excellence dans la pièce éponyme ${ }^{2}$.

2 Der Snob annonce par son titre une comédie "de caractère ", voire une satire du snobisme incarné, comme Le Bourgeois gentilhomme ou Les Précieuses ridicules donnent sans ambages le programme de la soirée. Mais Sternheim innove en ce sens qu'il donne une généalogie au snobisme et la poursuit parallèlement à l'histoire de l'Allemagne. Il ne serait donc pas hasardeux de voir dans la vie de Christian Maske une allégorie de la bourgeoisie allemande «snobifiée " par l'évolution particulière de l'Allemagne devenue « das deutsche Reich».

3 Point n'est besoin d'établir une chronologie vraisemblable de cette ascension dans et par le snobisme. À la fin de Die Hose, le père Maske, Theobald Maske, annonce qu'il va assurer sa descendance, avec son épouse Luise. Dans la pièce qui suit, celle que nous plaçons au centre de cette étude, le fils Maske acquiert à la fois un prénom (Christian), un âge (trente-six ans) et une situation sociale. De la même façon, Theobald a pris de l'âge (soixante ans), mais n'a guère changé de situation : il s'est hissé dans la carrière des fonctionnaires, sans atteindre un grade important. Fondamentalement, il est resté celui qui dit à la fin de Die Hose : "Je peux maintenant assumer la responsabilité de te faire un enfant. Que dis-tu? $»^{3}$ Il a en effet bien manœuvré pour pouvoir « assumer » : il a deux sous-locataires qui lui procurent une hausse de ses revenus. Die Hose est ainsi plutôt une comédie de situation, l'irrésistible ascension d'un modeste 
fonctionnaire besogneux et craintif, qui peut régner sans complexes sur son univers domestique, où sa femme a autant d'importance qu'un objet familier : le four, l'horloge, le vase à fleurs. Ce qui a évolué depuis le début de la pièce : les boutons-pression ont remplacé les rubans, empêchant toute chute intempestive d'une culotte de femme, vêtement qui provoque des fantasmes masculins.

Il faut avoir présente à l'esprit cette configuration primitive sans chercher à établir une histoire de la famille Maske. Sinon, les didascalies internes feraient naître le rejeton " assumé » du père Theobald et de sa femme Luise en... 1877. Tout se passe comme si une contraction du temps " gommait » une enfance et une formation se déroulant de la période dite Gründerzeit (avec Bismarck et le premier empereur Guillaume Ier) à l'émergence de l'impérialisme allemand, sous l'impulsion du second empereur, Guillaume II. Il faut prendre le personnage de Christian tel qu'il se définit dans son époque (1913), sans chercher à bâtir une vraisemblance dans le temps. Sa «vie antérieure " est dans un "temps avant", dont la cohérence importe peu. La scène d'introduction (I, 1) nous donne certes des détails sur ce «temps d'avant », mais ils ont pour fonction de contribuer à l'étiologie du snobisme de Christian, moteur de l'action.

Deux acquis le mettent en situation d'être snob. Il a vécu pendant quatre ans avec une maîtresse, Sybil, qui lui a appris « les bonnes manières ». Il s'est rendu très influent au sein d'une société par actions (AG), qui exploite des mines en Afrique. Il y possède même un cinquième des actions, et le conseil d'administration lui propose le poste de Generaldirektor. Cette situation sociale lui donne le devoir d'être à la hauteur. Tant pis pour Sybil, qu'il va remercier, aux deux sens du terme. Merci pour l'art de se vêtir, de se comporter. Mais il faut rompre, comme on le fait avec des relations d'affaires devenues inutiles. Pas de sentiments pour congédier une maîtresse. On solde les comptes : pour prix de ses bons et loyaux services, Sybil aura 24000 marks, et une rente mensuelle. Elle n'a pas le droit de se plaindre, c'est elle qui a engendré ce monstre froid. Son vocabulaire, il le lui doit :Abrechnung, Addition, Kalkül.

6 L'art de rompre avec une maîtresse est plus facile à exercer que celui de couper les ponts avec une famille "populaire », qui n'a pas pu lui enseigner « les rudiments de la vie en société ». Là commence le snobisme de Christian: quand on a une position sociale « obtenue de haute lutte ${ }^{4}$, il serait « de mauvais goût » de montrer « d'où on vient ». Le bon goût est symbolisé, à la fin de la scène de rupture, par la confection d'un nœud de cravate : le seul legs de Sybil.

7 Le nœud de cravate et le langage (écrit ou oral) vont de pair : ou bien « impeccable » ou bien «infâme ». Un monologue de Christian rappelle les hésitations de Monsieur Jourdain souhaitant rédiger un billet à l'adresse de la «belle marquise ». En l'occasion, c'est le comte Palen: comment répondre à son invitation «à dîner en tout petit comité $»^{5}$. Ses essais de vocabulaire autour du couple «impeccable» mais "significatif » sont un morceau de bravoure pour un comédien.

8 Cette recherche des mots adéquats poursuit Christian tout au long de la scène capitale où le fils arrivé s'efforce d'éloigner ses parents « populaires ». Sa pauvre mère Luise fait figure de prototype : un tailleur "impossible ", un chapeau " de travers », elle croit que "Generaldirektor » est un grade dans l'armée. Le marchandage ressemble à une partie de poker. Pour Theobald, le fait d'avoir élevé et éduqué donne droit à une reconnaissance éternelle. Christian met cartes sur table : je ne vous dois rien si je vous rembourse. Or j'ai établi une liste de vos dépenses. Cette liste est faite pour provoquer le rire méchant du spectateur. Tout y passe: frais d'entretien, de docteur, de 
pharmacie, d'habillement, de chaussures. Le total s'élève à 11800 marks, et Théobald accepte le marché, mais sur la base d'un malentendu : il croit que ce pactole lui servira à régler la pension du «bâtard » qu'il a engendré - nous le savons depuis les premiers mots de la pièce. Mais il y a une condition : pas question de venir vivre sa retraite près de son fils, a fortiori chez lui :

Ma vie est devant un tournant décisif. J'ai absolument besoin, surtout pour mon avenir immédiat, d'être libre de toutes considérations familiales ${ }^{6}$.

Il s'ensuit une explosion de colère chez Theobald qui culmine dans la réplique : « $\mathrm{Si}$ j'avais su ça, on t'aurait noyé dans ton premier bain ». Mais il ne faut pas oublier que Sternheim est avant tout un auteur de comédies, ce qui explique l'évolution de la querelle familiale vers une vision grotesque : l'éloignement des parents conçu comme un voyage d'agrément choisi par consultation d'un guide Baedeker. La simple lecture de ce guide provoque l'hilarité, renforcée par les commentaires niais de Theobald. Bruxelles ne lui convient pas, car un Allemand de bon aloi ne saurait suivre des « coutumes étrangères (welsche) $»^{7}$. Alors Zurich ? Au moins, on y parle allemand. C'est la solution: Christian parle comme une agence de voyages, promet un complément de pension pour convaincre le client. L'affaire est à saisir tout de suite, car elle permettra à Christian de se donner à son combat " pour une place bien visible dans la vie $»^{8}$. Il y a de quoi être étonné : le fils écrase le père en adoptant la rhétorique du combat pour la vie. Dans le contexte, cela donne une bataille de chiffres: Theobald s'incline devant la montée des enchères.

Le snobisme figurera dans l'arsenal des moyens pour "arriver». Cela signifie le rapprochement avec la noblesse, qui se fera par étapes. Pour commencer, pas d'empressement, mais de la réserve. Christian est satisfait de sa réponse dilatoire à l'invitation du comte Palen: "La multiplicité des affaires, cher Comte Palen, m'empêche hélas d'accepter votre aimable invitation $»^{9}$. Mais il se trompe sur la tactique. C'est la noblesse qui veut l'intégrer dans son corps: la Société des mines de Monambo a un Conseil de surveillance composé uniquement de nobles. Le comte en est l'émanation et a pour tâche de faire subir un « examen de passage » à Christian avant de le coopter comme Generaldirektor.

11 L'impétrant est suffisamment préparé pour répondre au discours du comte par un discours compatible. Mais le comique s'insinue dans cet échange verbal: aux mots délibérément vagues du comte, le brillant sujet répond dans les mêmes termes. Le comte recherche "les mêmes convictions", "l'appartenance à notre cercle par toute une conception de l'existence ", "la sensibilité aux valeurs du goût supérieur». Le discours de Christian est gestuel et laconique : «il s'incline » devant cet adoubement.

Le comte n'hésite pas à bâtir une légende autour de Christian («vous êtes d'une excellente famille », "une éducation parfaite»), tout en étant attaché à des postures superficielles, comme « le port d'une cravate ». Ce qui fait de lui un snob à son tour. On l'entend défendre "l'acceptabilité » d'un «bourgeois » à partir des "actions de ses ancêtres ». Or, on ne sait rien de Christian dans les «bons cercles », et pour lui cela vaut bien mieux. Il prétend que ses parents sont «morts, tous morts». Il serait en quelque sorte pur et vierge, après ce reniement de Pierre, et prêt à reconnaître, comme s'il était adopté, le principe de supériorité du porteur d'un « diplôme de noblesse ». Ravissement du comte: vous m'avez convaincu, je peux vous offrir ma garantie. Christian ne peut plus refuser l'invitation à dîner. Contemplant un nœud de cravate, il a la même 
réaction que Schippel, à la fin de la pièce éponyme («Du bist Bürger, Paul !: Das ist erreicht $»)$.

Tout ne va pas pour le mieux dans la famille von Palen, et la richesse de Christian tombe à point nommé pour la sortir de « conditions difficiles». À condition que la fille de la maison, Marianne, accepte de l'épouser. Elle n'y est pas opposée, elle sent en lui une «force naturelle» qui emporte tous les obstacles. Mais le fait qu'il soit un « roturier» (le qualificatif «bürgerlich» semble posséder le même poids que dans le théâtre de Lenz et du jeune Schiller) est tout de même l'obstacle par excellence ${ }^{10}$. Christian aura beau accepter de vivre «selon les lois » de la noblesse, il lui manquera toujours la goutte de sang bleu dans les veines qui fait le partage. Le comte Palen compare ce sang bleu au gazon du duc du Devonshire: des siècles d'entretien le rendent incomparable. La noblesse ne s'acquiert pas, elle est là depuis toujours, on la reçoit à sa naissance, on ne peut l'emprunter ${ }^{11}$.

C'est bien là que l'on peut parler d'une comédie historique, qui procède d'une situation : une classe qui monte dans l'échelle sociale et politique, une classe qui essaye de se maintenir en haut, ou du moins de sauver les apparences. Nous sommes dans l'époque wilhelminienne encore splendide, Berlin voulant une grande et belle place au soleil. Pour cela, il faut une alliance entre l'aristocratie et la bourgeoisie industrielle. Mais attention : chacun dans son rôle. À Christian, les rencontres avec «le Ministre des colonies ", la présentation solennelle à "Sa Majesté $»^{12}$. Au comte Palen, la gestion avisée de son "capital prestige». Pour le dire tout net: à chacun son signifiant. Christian en fournit deux : l'équitation et les tableaux de maître. Arriver chez soi en costume de cavalier, c'est le premier grade dans la hiérarchie des tenues, qui culmine avec les tenues militaires. Par contagion sémiologique, le fait de chevaucher une belle bête, issue d'une bonne écurie, équivaut à s'intégrer à la noblesse... sur le dos d'un cheval. De même, la possession d'un Corot vous introduit dans le monde réputé supérieur des collectionneurs. On se fait ainsi une galerie des ancêtres. Pour sa part, le comte Palen déploie un signifiant imparable: son inscription dans le Gothaer Almanach $^{13}$ : «Palen. Très vieille noblesse westphalienne, mentionnée pour la première fois en 1220 avec un Rutger Palen ». Le vocabulaire de la sélection par le sang, selon les règles d'un pedigree, est identique à celui d'un Hard-book. Augustus Aloysius a épousé Elisabeth Gräfin von Fürstenbusch, il a vécu dans un domaine (" auf Ernegg »), il a engendré deux enfants qui sont ses « rejetons » («Sproß») ${ }^{14}$.

Qui va l'emporter dans cette course entre snobs, celui qui monte ou celui qui descend? La lutte est triviale comme une partie de cartes. La carte maîtresse de l'un : "Comte Augustus von Palen, je vous demande la main de votre fille Marianne». La carte du comte Palen est attendue : ce sont des "préjugés de caste " (Standesvorurteile) qui lui font refuser cette union. Paradoxalement, ce refus de principe comble Christian, qui lui dit en substance: je n'en attendais pas moins de vous. Il fait même l'éloge de la «fermeture par l'intérieur $»^{15}$ de l'état nobiliaire, qui se protège en employant moult vocables français germanisés : emballiert, attachiert. Sous des apparences bénignes, le débat est âpre. Votre noblesse sent l'écurie, le haras. Votre roture vous rend anonyme, alors que nous vivons sous le regard constant de "nos lignées », et que nous devons renoncer de ce fait aux «jouissances d'une vie débridée au sein d'une masse sans identité $»^{16}$.

16 Quadrature du cercle : pour entrer dans la « communauté » nobiliaire, il faut bien qu'un nom ouvre la voie et amasse ensuite des générations irréprochables à travers les 
siècles. Les souverains résoudront le problème en "créant " une noblesse bourgeoise (bel oxymore : noblesse de robe, noblesse d'Empire). Christian est prêt à s'engouffrer dans la brèche : mes parents et grands-parents furent « tous fonctionnaires au service de l'État ». Deux mots sésames. D'ailleurs, l'Empereur lui-même vient de récompenser un roturier en le nommant Ministre des Postes. La partie de cartes se termine par une sorte de capitulation du comte: ma fille sera à vous, mais sans dot, car nous avons perdu presque toute notre fortune, et ce qui reste couvre les droits exigés par le régiment (d'élite bien entendu) où sert notre fils.

On peut affirmer dès lors que la messe est dite. Le comte snob, en avouant son impécuniosité, a perdu tous ses droits à la morgue nobiliaire. Donc les géniteurs de Christian sont devenus présentables, et de préférence sans attendre. Un peu de mises en garde, et ils feront des parents tout à fait convenables. Qui plus est : en les associant à son triomphe, Christian tiendra une revanche. Une autre façon d'être snob se fait jour en lui : celui d'être un vainqueur dans les «combats pour l'existence». Une ultime rencontre avec son ex-maitresse Sybil lui permet de déverser sur elle son darwinisme primaire, qu'il oppose aux « grandes phrases de la social-démocratie », dont elle serait porteuse. Scène de rupture assez violente: Christian se voit reprocher d'« adorer » ses parents, après les avoir brûlés. Un snobisme inversé, qui fait chic, impose que l'on ne cache pas ses origines dès lors que l'on a gagné assez d'argent. Mais Christian ne va pas jusqu'au bout de sa nouvelle logique : il devrait imposer aussi son ancienne maîtresse, qui l'a soutenu à ses débuts à coups de billets de vingt marks. Menace : si elle venait à le divulguer, il l'accuserait de diffamation devant les tribunaux.

Le père Maske, arrivé inopinément chez son fils en raison de la mort subite de sa mère Luise, désapprouve Christian quand il apprend ses «fiançailles » avec une comtesse : c'est une folie, presque un rapt. Et moi qui n'ai jamais vu un comte dans ma vie, je vais être tourné en ridicule. C'est comme si le père de George Dandin tançait son fils: "marier une souris avec une girafe, danser sur une corde raide $»^{17}$. Une sorte de snobisme à l'envers: le bon vieux Theobald n'a rien à faire avec une ribambelle de comtes et comtesses. C'est la morale du chacun chez soi, du chacun son métier, pour vivre heureux, vivons cachés. La palinodie de Christian ("que tu viennes vivre chez moi ») provoque l'indignation du père Theobald, qui veut rester « homme du peuple», un point c'est tout. J'ai ma dignité, tu veux me l'ôter, comme vengeance envers mon éducation rigoureuse.

19 Une palinodie chasse l'autre. L'entrée surprise du comte crée une situation comique : Theobald va se trouver charmé par les bonnes manières de l'aristocrate détesté. Toujours à l'aise, on sait vivre, on s'adapte. Christian n'était donc pas orphelin, c'est tout simplement «charmant». Sa mère est morte, le père est veuf, on lui donne du «très cher monsieur ». En toute simplicité, on le convie à un petit déjeuner, avec un verre de bon vin et l'emploi de termes familiers : c'est pour mieux se «sentir» (en confiance). En un mot, Theobald est séduit par le comte comme Zerline par Don Giovanni.

20 Christian y flaire un piège : le comte veut exploiter la situation pour compenser son aveu de faiblesse... pécuniaire. J'ai voulu "renier » mon père, c'est ma très grande faute, on va en faire des gorges chaudes dans la famille de Marianne, pourtant heureuse d'être demandée en mariage par ce brillant sujet. Quelle stratégie adopter? Pas moyen de se défaire de ce père encombrant, qui risque de commettre impair sur impair. Il 
aurait mieux valu avouer d'emblée mes humbles origines. Donc, ne pas me conduire en «snob».

21 Le troisième acte fait partie de ces passages de comédie où la cérémonie des noces fournit l'occasion de mettre à nu l'hypocrisie des comportements et le ridicule des participants. Comme cette " grosse comtesse » qui mange salement et ce chevalier qui a une face de grenouille. Christian est dûment marié, mais il soupçonne que ce sacrement a été accueilli par des railleries, dont témoigne une conversation entre Marianne et sa femme de chambre, ponctuée de rires et de chuchotements. Il capte des bribes de ce dialogue et s'imagine (non sans raison) qu'on se moque de lui à cause de ses "moustaches en croc", que la domestique, avec l'assentiment de sa propre épouse, trouve ridicules et hilarantes ${ }^{18}$.

22 A-t-il conclu un marché de dupes? Si la vie est un combat, à qui profite l'opération? Pour Theobald, que l'ivresse rend bavard (et vulgaire), ce mariage est une revanche, une remise en cause absolue du principe de révérence "Schuster, bleib bei deinem Leisten ». Il célèbre son "sang de roturier ", qui est arrivé "à mettre dans son lit une petite poulette aristocratique $»^{19}$. Il en oublie que son fils avait voulu le renier, l'installer en exil à Zurich, le faire disparaître de la vue de son nouvel entourage aristocratique. La nouvelle aristocratie, c'est la dynastie Maske, qui peut s'imposer en «desserrant sa ceinture $»^{20}$. Les vieux nobles auront beau se moquer lourdement des lapsus du père (il a confondu l'Ordre de l'Aigle des Hohenzollern avec la Croix de Fer), ils ne comprennent pas quelle est leur situation. Le vocabulaire martial s'insinue dans la célébration commune d'une "aurore " de la «lignée » des Maske; tendre l'arc, lancer une flèche, s'agripper aux remparts, humiliation de l'ennemi, qui s'incline devant le vainqueur ${ }^{21}$.

On ne peut pas parler de véritable roman d'amour entre Marianne et son époux Christian. Conformément aux usages, l'époux ne voit sa femme dévêtue que le soir de ses noces. Jusque là, ils sont étrangers. Ils font connaissance dans l'épisode du coucher. Marianne accumule les clichés: je suis curieuse de savoir combien de femmes tu as aimées, moi je n'ai eu qu'un flirt avec un petit aspirant, je n'avais que seize ans. Pas beaucoup d'érotisme dans cette scène cruciale. C'est l'union d'une petite aristocrate avec un homme de trente-six ans qui ne porte aucun titre, sauf: Directeur général de notre plus grande firme nationale, $20 \%$ du produit national brut. Cela n'a pas l'air d'exciter furieusement la jeune épouse, surtout quand son mari lui fait un cours de management. Elle se donne du mal pourtant, ses bras autour de son cou, assise sur ses genoux. Mais rien n'y fait : ce qui excite le « capitaine » (d'industrie), c'est le capital en actions, la société industrielle, l'économie politique. Pas la richesse en elle-même, mais le pouvoir d'influer sur le destin de millions d'Allemands.

24 Ce capitaine d'industrie peut passer de la paranoïa (les aristocrates, ces snobs, se moquent de moi en cachette) à la mégalomanie. Il se voit comme le descendant éminent d'une " race » aux qualités particulières, qui fait encore mieux que son père, et aussi son grand-père. La préparation de la nuit de noces consiste pour les époux à exposer leurs beautés et à les comparer. Marianne a son diadème de marquise, qui lui vient d'une arrière grande-tante. Christian n'a que des beautés postiches : un portrait de sa mère peint par... Renoir et surtout une généalogie éblouissante où tout est inventé. C'est le comique du mensonge sur les origines.

25 La fameuse Luise de La Culotte serait issue d'une lignée de chefs vikings, qui aurait approché le roi de Suède Charles XII. Pour qui a suivi dans la pièce précédente la 
véritable histoire de la vraie culotte, cette fabrication d'une légende est hilarante. La brave Luise devient une dame au Bois de Boulogne, remarquée par Renoir en personne, accompagné par un vicomte français. Christian fait passer une copie d'un tableau de Renoir pour un portrait de sa mère. Marianne, sans soupçons, gobe le mensonge, auquel Christian se met à croire lui-même, tout en déshabillant la jeune épouse. Il porte l'estocade finale en laissant entendre que le vicomte aurait séduit prestement la bonne Luise dans un pavillon du Bois. La petite poule aristocratique en tombe en pâmoison: "J'ai l'impression que le dernier mur entre nous s'effondre, comme si je pouvais désormais me fondre en toi sans réticence $»^{22}$. Dernière didascalie : Marianne tombe aux pieds de son « mari et maître ». Le snob a dû devenir mythomane pour se fondre à son tour dans la caste nobiliaire.

Le but de la comédie, donc de l'auteur comique, est depuis toujours de corriger les mœurs de son temps en faisant rire aux dépens de ceux qui les incarnent ${ }^{23}$. Le snobisme est multiforme dans la pièce de Sternheim, et (presque) tous les personnages en sont atteints. La scène finale est à cet égard exemplaire. La jeune comtesse Marianne se trouve placée dans la posture ridicule de "gober » un mensonge éhonté, massif et égrillard : le récit de l'adultère champêtre commis par la pauvre Luise, recevant sa part de sang bleu du vicomte anonyme, français sans aucun doute, et la transmettant à son fils Christian, qui s'en prévaut trente-six ans plus tard. Il y a lieu de penser que sa défloration, largement annoncée et avancée par son déshabillage, en acquerra un « supplément » de plaisir. Le snobisme de Marianne la confie à l'étreinte d'un escroc: bien fait pour elle.

Christian Maske (nomen est omen), le concepteur de la superbe mise en scène prénuptiale, fondée sur la présence d'un faux tableau de Renoir, semble trouver plutôt son plaisir dans la jouissance mentale de ses capacités d'économiste : tenir des propos " techniques » à sa jeune épousée au soir fatidique des noces ne préjuge en rien de la qualité justement aussi «technique » du mari, qui se doit de réussir la première union comme il a réussi sa carrière. Son langage abstrait n'a rien d'érotique, et les bâillements d'ennui et de fatigue de Marianne le désignent à l'hilarité publique. Le snobisme satisfait de Christian ne le transforme pas en jeune premier rayonnant qui épouse la femme de sa vie.

On émettra l'hypothèse d'une «allégorisation » du personnage titre : le snob Christian serait la figuration, la personnalisation de l'Allemagne wilhelminienne dans sa volonté d'accéder au statut de la Weltmacht: il manquait à ce Reich allemand ce qui appartenait sans conteste à la France et à la Grande-Bretagne. La personne de Guillaume II, avec sa jeunesse relative en arrivant au pouvoir (vingt-neuf ans), incarne bien ce saut qualitatif largement préparé par l'ère Bismarck, bien que le chancelier de fer ait pensé avant tout à une suprématie européenne de l'Allemagne.

Un très intéressant recueil d'images de la période 1888-1918 peut se regarder en liaison avec les comportements de ce snob de Christian ${ }^{24}$. On y trouve d'abord la construction d'une légende du Reich, qui fait oublier les siècles de morcellement des terres allemandes. Elle commence avec Barbarossa, le fondateur, dont le culte est organisé par la construction d'un monument massif au Kyffhäuserberg (en 1894). Il faut ensuite sauter au "grand Frédéric» du xviIIe siècle et à la reine Louise de Prusse de l'occupation napoléonienne pour garder le fil de la légende. Le Reich "rattrape » son retard à force de constructions: le château kitsch des Hohenzollern dans le fief d'origine, l'invention de l'allégorie Germania (Niederwalddenkmal). 
La seconde série d'images renvoie aux propos «économiques » de Christian, avec leur sommet dans la chambre nuptiale. L'une d'elles annonce fièrement que dans les deux décennies qui entourent 1900 la population a cru de $39 \%$ et le nombre de porcs de $75 \%$. Une carte " patriotique » montre un respectable chêne abritant la population en 1907. Rien que pour les "grandes entreprises", elle est passée de 1,6 millions en 1882 à 5,3 millions. Dans ce contexte, la présence du Reich en Afrique est illustrée par des cartes du monde, éditées par un "Verein für das Deutschtum im Ausland».

Le Kaiser est omniprésent dans ce recueil, portant les uniformes les plus variés, souvent en amiral. Une seule constante: les moustaches relevées en pointe. Ces moustaches impériales, que Christian affiche au banquet de ses noces, annoncent avant le tournant du siècle l'ère nouvelle de la Weltmacht (conditionnée par une Weltpolitik). Les visages barbus de la célèbre proclamation de l'Empire allemand à Versailles en 1871 se sont effacés devant le visage glabre et superbement orné de belles moustaches.

On peut considérer que les deux premières comédies de la tétralogie sur la famille Maske constituent ce qu'il y a de plus durable dans la satire des mœurs au fil de l'évolution de la société allemande. Les statistiques des mises en scène en font état ${ }^{25}$. Dans 1913, le désormais vieux Christian Maske (il a soixante-dix ans, il est à la tête d'un Konzern de l'armement, et s'appelle baron von Buchow, du nom d'une propriété déjà achetée au temps où il était «snob») constate que ses rejetons (nés du mariage avec la comtesse Marianne) n'ont pas sa morale de l'entreprise. Ils ne pensent qu'à profiter d'une situation qu'ils n'ont pas acquise, et l'aînée Sophie essaie même d'évincer son père de la conduite des affaires.

Dans Le Fossile (1921-22), le chef de la dynastie Maske a disparu, et le flambeau de la « vraie » noblesse est tenu par un vieux général, Traugott von Beeskow, beau-père de Sophie. Il est quasiment gâteux, montré sur un cheval à bascule, sabre au clair, célébrant l'anniversaire de l'ex-empereur. La classe nobiliaire est devenue pour une grande part une classe fossile, et les efforts de Christian Maske dans Le Snob, bien que frappés par le ridicule, apparaissent bien vains et presque dignes de compassion.

\section{NOTES}

1. Il s'agit là de l'année de l'écriture, l'année de la création pouvant être différente. Les pièces de Sternheim concernées sont dans l'unique édition du Gesamtwerk, par Wilhelm Emrich, en 10 volumes, Luchterhand, Neuwied, 1963-1976. Le théâtre occupe le volume 1. Nous citerons sous le sigle GW 1.

2. Si l'on se fie au dictionnaire Le Robert, le mot provient de l'argot des étudiants de Cambridge, où il désigne, en 1857, un « cordonnier ", plus largement celui qui n'est pas de l'université. Sera désignée sous ce nom «toute personne qui cherche à être assimilée aux gens distingués de la haute société en faisant étalage des manières, des goûts, des modes qu'elle lui emprunte sans discernement et sans besoin profond, ainsi que des relations qu'elle peut y avoir ». Mais dans cet ensemble, la personne de la haute société qui désigne les snobs qui la singent peut être taxée 
aussi de snobisme en affirmant ostensiblement sa différence. Il y aurait ainsi deux snobismes : celui de l'assimilation et celui de la distinction.

3. Il n'existe qu'une seule traduction (partielle) du théâtre de Sternheim : le recueil Scènes de la vie héroïque des bourgeois (texte français de Catherine Gicquel-Bourlet, préface de Jean-Claude François), L'Harmattan, Paris, 2009. Il comprend trois pièces : La Culotte, La Cassette, Schippel le Bourgeois. Dans cette édition, cf. la scène finale, p. 119.

4. Der Snob n'étant pas traduit, force est de se référer au texte allemand du Gesamtwerk. Les citations sont donc de notre plume, avec éventuellement le texte original en bas de page. Ici : GW 1, p. 143, "errungene Stellung». Dans ce contexte, Sybil se lance dans un jeu de mot involontaire : «du hast wie ein Neger gearbeitet », adressé à celui qui s'occupe de mines... en Afrique.

5. En français dans le texte allemand. L'usage du français par le comte Palen est la marque du snobisme de distinction (cf. GW 1, p. 146).

6. GW 1, p. 155 : "Mein Leben steht vor vollkommener Wendung. Ich muß, für die nächste Zeit vor allem, von verwandtschaftlichen Rücksichten frei sein".

7. GW 1, p. 157. Comment traduire cet adjectif «typiquement » allemand, qui décrit et décrie une certaine « romanité »?

8. On ne peut manquer de penser à la formule «Wir verlangen auch unseren Platz an der Sonne ", lancée par le chancelier von Bülow en 1897. GW 1, p. 159: "Der Kampf um eine sichtbare Stelle im Leben ist gewaltig ".

9. GW 1, p. 161: "Mannigfaltigkeit der Geschäfte, verehrter Graf Palen, verhindert mich leider, Ihre liebenswürdige Einladung anzunehmen".

10. Citons pour Lenz : Der Hofmeister (1774) et Die Soldaten (1775), et pour Schiller : Kabale und Liebe (1783). En France, le mot roturier désignait les non-nobles du tiers état, même s'ils étaient par ailleurs plus riches que les gens «à particule " (comme certains bourgeois). À l'origine, le mot roture désignait la redevance due au seigneur pour une terre à défricher, on disait qu'un fief «tombait en roture ». En Allemagne, qui ne connaît que le mot bürgerlich, les deux notions sont associées, roturier et bourgeois.

11. La noblesse est liée à l'origine à la possession de domaines, parfois grands, en Prusse, Westphalie, Bavière. Mais la baisse des prix agricoles lamine les revenus de la noblesse rurale. Très souvent, elle tire des revenus de sa présence privilégiée dans l'appareil d'État : « entre 1888 et $1914,70 \%$ des ministres prussiens, $64 \%$ des généraux, $62 \%$ des diplomates, $57 \%$ des hauts fonctionnaires appartiennent à la noblesse » (cf. François Roth, L'Allemagne de 1815 à 1918, Armand Colin, Paris, 1996, p.124). Un certain nombre d'industriels furent anoblis (Krupp, Siemens, Borsig). Dans le cas Krupp, c'est une héritière, la fameuse Bertha, qui devient baronne von Bohlen und Halbach.

12. Les principales colonies d'Allemagne sont en Afrique: Togo, Cameroun, Südwestafrika (aujourd'hui : Namibie), et Ostafrika. Elles n'auront qu'une brève existence : de 1884 à 1918.

13. La petite capitale du petit duché de Sachsen-Coburg-Gotha a édité, de 1764 à 1945, un almanach établissant la généalogie des grandes familles nobles d'Europe. C'est devenu et resté un nom commun.

14. Le mot Sproß convient aussi bien au règne végétal et animal qu'au règne... nobiliaire (cf. GW 1, p. 173).

15. GW 1, p. 174 : «Eben diese innerliche Abgeschlossenheit ist eine Eigenschaft Ihrer Kreise ».

16. GW 1, p. 175 : «Verzicht auf Wollüste eines freien Lebens in namenloser Masse ».

17. GW 1, p. 186 : «Die Maus mit der Giraffe willst du verkuppeln, Seiltänzerstücke machen ».

18. GW 1, p. 198. On n'entend que ces bribes de la conversation, séparées par des rires francs: "Schnurrbartspitze... ein bißchen lächerlich... es war zu komisch...»

19. GW 1, p. 201: «Wie du dir so ein adeliges Hühnchen ins Bett holst, das brachte mein Bürgerblut zum Sausen ». 
20. Ibid. : «In dir ist alles Maskesche um ein paar Löcher weitergeschnallt ».

21. Les didascalies sont éloquentes: (Theobald) umarmt ihn..., beide kichern und fallen sich in die Arme. (GW 1, p. 201, p. 203).

22. GW 1, p. 214 : « Und mir, als ob doch eine letzte Wand zwischen uns niederfällt, als ob erst jetzt ich ungehemmt in dich versänke ».

23. La célèbre formule "castigat ridendo mores » (c'est en riant que l'on châtie les mœurs) n'aurait été forgée qu'au XVII ${ }^{\mathrm{e}}$ siècle, mais elle existait déjà sous d'autres formes dans la littérature latine.

24. Ansichtskarten und Texte aus der wilhelminischen Zeit, 1888-1918, éd. par Wilhelm Stöckle, Ernst Klett Verlag, Stuttgart, 1972 ( $1^{\text {ère }}$ édition).

25. Les statistiques portant sur la période 1947-1975 donnent une idée de l'enracinement de Sternheim dans le répertoire allemand. Le classement se fonde sur le nombre de mises en scène : La Culotte (63), Le Bourgeois Schippel (55), La Cassette (54), Le Snob (44). L'écart se creuse avec Tabula rasa (21). Aucune indication indication sur les autres pièces de la tétralogie. Cf. Was spielten die Theater? Bilanz der Spielpläne in der Bundesrepublik Deutschland 1947-1975, éditions du Deutscher Bühnenverein Köln, 1978. Dans cette publication, Sternheim occupe la $16^{\mathrm{e}}$ place au répertoire mondial (entre Ibsen et Goldoni), et il est le $10^{\mathrm{e}}$ des auteurs allemands (entre Dürrenmatt et Thoma).

\section{RÉSUMÉS}

La comédie de Carl Sternheim Der Snob (1913) fait partie d'une tétralogie consacrée à l'ascension et à la ruine de la famille Maske. Elle prend la suite immédiate de Die Hose (1912) : le fils (Christian) du fonctionnaire petit-bourgeois (Theobald) est devenu directeur d'une grande entreprise minière et veut s'intégrer à la classe nobiliaire par son mariage avec une jeune comtesse, Marianne von Palen. Mais il lui faut vaincre les réticences du père, qui reconnaît cependant ses mérites. Alors il singe les comportements de la noblesse, pratique l'équitation et l'escrime, soigne sa mise et son langage. Il fait sauter le dernier verrou en faisant croire qu'il est issu d'un adultère entre sa mère (Luise) et un vicomte français. C'est une charge comique contre les valeurs de la société allemande wilhelminienne précédant la guerre de 1914-1918.

Carl Sternheims Komödie Der Snob (1913) ist Bestandteil einer Tetralogie über Aufstieg und Fall der Familie Maske. Sie ist die unmittelbare Folge des Stückes Die Hose (1912) : Der Sohn (Christian) des kleinbürgerlichen Staatsbeamten Theobald ist zum Direktor eines mächtigen Bergbauunternehmens geworden und will durch die Heirat mit einer jungen Gräfin, Marianne von Palen, in den Adelsstand aufsteigen. Er muß aber den Widerstand des Vaters überwinden, der immerhin seine Verdienste anerkennt. Um sein Ziel zu erreichen, äfft er das adelige Verhalten nach, übt sich in der Reit- und Fechtkunst, pflegt Kleidung und Wortschatz Die letzte Hürde überspringt er, als er sich als der illegitimene Sohn eines Ehebruchs seiner Mutter (Luise) mit einem französischen Vicomte ausgibt. Im Ganzen gerät die Handlung zu einem lustigen Angriff auf die Werte der wilhelminischen Gesellschaft im Vorfeld des Ersten Weltkriegs.

Carl Sternheim's comedy Der Snob (1913, is the second part of a tetralogy devoted to the social ascent and fall of the Maske family - and thus the continuation of Die Hose (1912).Christian, the son of Theobald, a lower middle class civil servant, has become the head of a large mining 
company and wants to join the nobility by marrying a young countess, Marianne von Palen. But he has to break through the defences of a father who nonetheless acknowledges his abilities. Therefore he apes the behaviour of a nobleman and starts practising horse riding and fencing, adopts an elegant dress-style and the appropriate manner of speaking. He finally manages to settle the questions about his decent by pretending to be the fruit of an adulterous relationship between Luise, his mother, and a French viscount. The play is caricature portrait of the manners and values of the Wilhelminian German society just before World War I.

INDEX

oeuvrecitee Der Snob

\section{AUTEURS}

\section{JEAN-CLAUDE FRANÇOIS}

Professeur émérite, Université de Nantes 